This item was submitted to Loughborough's Research Repository by the author.

Items in Figshare are protected by copyright, with all rights reserved, unless otherwise indicated.

\title{
A case study of Twitter as a research tool
}

\section{PLEASE CITE THE PUBLISHED VERSION}

https://doi.org/10.1080/17430437.2017.1342622

\section{PUBLISHER}

(c) Taylor \& Francis

\section{VERSION}

VoR (Version of Record)

\section{PUBLISHER STATEMENT}

This work is made available according to the conditions of the Creative Commons Attribution-NonCommercialNoDerivatives 4.0 International (CC BY-NC-ND 4.0) licence. Full details of this licence are available at: https://creativecommons.org/licenses/by-nc-nd/4.0/

\section{LICENCE}

CC BY-NC-ND 4.0

\section{REPOSITORY RECORD}

Corbett, Ben, and Allan Edwards. 2019. "A Case Study of Twitter as a Research Tool”. figshare. https://hdl.handle.net/2134/32403. 


\section{A case study of Twitter as a research tool}

\section{Ben Corbett \& Allan Edwards}

To cite this article: Ben Corbett \& Allan Edwards (2018) A case study of Twitter as a research tool, Sport in Society, 21:2, 394-412, DOI: 10.1080/17430437.2017.1342622

To link to this article: https://doi.org/10.1080/17430437.2017.1342622

\section{册 Published online: 07 Aug 2017.}

Submit your article to this journal 2

\section{Шll Article views: 156}

Q View related articles $\sqsubset$

View Crossmark data $\nearrow$ 


\title{
A case study of Twitter as a research tool
}

\author{
Ben Corbett ${ }^{\mathrm{a}}$ and Allan Edwards ${ }^{\mathrm{b}}$ (I) \\ aInstitute for Sport Business, Loughborough University London, London, UK; ${ }^{\mathrm{b}}$ Discipline of Sport and Exercise \\ Science, Faculty of Health, University of Canberra, Canberra, Australia
}

\begin{abstract}
This paper documents the process of utilizing Twitter for a doctoral dissertation in rugby governance, and highlights the key benefits of using Twitter as a research tool. Research followed a case study approach to delve into the four major uses of Twitter: (1) a warning system for dramas or incidents relating to one's research field; (2) data collection; (3) finding and recruiting research subjects; and (4) building a personal research brand. The research design used a mixed method approach that combined qualitative and quantitative analysis methods. The findings serve as the basis for the pursuit of more detailed future research with Twitter as the principal medium in focus within the area of sport.
\end{abstract}

\section{Introduction}

The study of Web 2.0 technologies increases with the rapid spread of Internet technologies and communications. Web 2.0 encompasses the various segments of social media such as blogs, social networking sites (SNSs) (e.g. Facebook, Twitter), content communities (e.g. YouTube), team websites, forums and bulletin boards. While the underlying functionality of social media sites is similar, the way the sites function and the type of users they attract can be drastically different (Lipsman 2009). Some SNSs focus on users and their life (e.g. Facebook), some focus on the message (e.g. Twitter), some focus on collecting and discovering ideas (e.g. Pinterest), some focus on knowledge creation (e.g. Quora), while some other focus on pictures (e.g. Instagram), or finding jobs (e.g. LinkedIn). Since the activities often drastically differ, different networks present different opportunities and challenges for research (Constantinides and Fountain 2008; Ioakimidis 2010; Li, MacIntosh, and Bravo 2012; Williams and Chinn 2010).

Facebook and Twitter are the most utilized sites for both sharing and accessing news and updates on the unfolding events. Research shows that due to the 'walled garden' approach, Facebook has become less accessible than Twitter for public communication Bruns and Stieglitz (2012). Given Twitter updates are publicly available to non-registered users, it is a platform that allows any user to follow any other user without knowing them personally.

This paper documents the process of utilizing Twitter for a doctoral dissertation in rugby governance, and highlights the key benefits of using Twitter as a research tool. The original

CONTACT Ben Corbett $\otimes$ b.d.corbett@lboro.ac.uk 
doctoral research explored organizational change occurring within rugby national governing bodies in response to Olympic inclusion of the short form or rugby called 'Sevens' (Corbett et al. 2015). The personal research brand, established mainly though Twitter, provided the clout and networks for a postdoctoral investigation of Sevens high performance ecosystems funded by rugby's international federation, World Rugby (Corbett, O’Brien, and Gowthorp 2015; O’Brien, Corbett, and Gowthorp 2015).

Research follows a case study approach to delve into the four (4) major uses of Twitter in the sport of Sevens:

(1) a warning system for dramas or incidents relating to one's research field;

(2) data collection;

(3) finding and recruiting research subjects; and

(4) building a personal research brand.

\section{Why Twitter?}

This paper primarily focuses on Twitter data for the following reasons: although many social media corpora are available, the data scale and accessibility is not comparable to Twitter data. Another reason for our interest in Twitter data is its many unique characteristics discussed below.

\section{Twitter}

Twitter is a free social networking website, launched by Jack Dorsey in 2006, that allows users to create an account using a 'handle' or unique username. Twitter propositions account holders to 'connect with people, express yourself, and discover what's happening' (Twitter 2016). This micro-blogging service allows account holders to post and read small messages of 140 characters or less, and to share photos, six-second video clips via Vine, live video via Periscope and links to other websites.

An important aspect of Twitter is that anyone with access to it can express their views, and although some users have more influence than others, 'a trend can be initiated by anyone, and if the environment is right, it will spread' (Cha et al. 2010a, 11). One attribute of the Twitter environment is competition for attention, which Romero et al. (2011) describe as 'ideas, opinions, and products compete with all other content for the scarce attention of the user community' (1). They go on to say 'in spite of the seemingly chaotic fashion with which all these interactions take place, certain topics manage to get an inordinate amount of attention, thus bubbling to the top in terms of popularity and contributing to new trends and to the public agenda of the community' (1). Sevens is one particular community that meets this challenge through Twitter. Sevens was chosen as the area of study as it was of particular interest to both authors. World Rugby showed particular interest in this research and provided both financial and service support to this research.

\section{Twitter uptake and usage}

Twitter emerged as a top-10 trafficked website by 2012 and continued to be in the top-10 in 2016. Usage statistics can be seen in Table 1. Its popularity pressured almost all sports 
Table 1. Twitter usage statistics as of October 2016.

\begin{tabular}{ll}
\hline Twitter company statistics & Data \\
\hline Total number of registered Twitter accounts & 696 million \\
Total number of active Twitter users & 342 million \\
Number of active Twitter users every month & 115 million \\
Number of new Twitter users signing up everyday & 135,000 \\
Number of unique Twitter site visitors every month & 195 million \\
Average number of tweets per day & 58 million \\
Number of Twitter search engine queries every day & 2.1 billion \\
Percent of Twitter users who use their phone to tweet & $43 \%$ \\
Percent of tweets that come from third-party applicants & $60 \%$ \\
Number of people that are employed by Twitter & 2500 \\
Percent of Twitters who don't tweet but watch other people tweet & $40 \%$ \\
Number of days it takes for 1 billion tweets & 5 days \\
Number of tweets that happen every second & 9100 \\
\hline
\end{tabular}

Sourced from http://www.statisticbrain.com/twitter-statistics on 1 October 2016.

entities to create an account to share information and market themselves. For example, and relevant to the case study, 132 of 152 rugby national governing bodies (that could be identified by searching Twitter) created an account by 2016, as have thousands of individual Sevens administrators, coaches, athletes and media agencies.

The emergence of third-party mobile applications (such as Twitter) on mobile devices (tablets and smart phones) has increased the pathways in which sport follower can consume sport, thereby forcing sport organizations to adopt social media such as Facebook and Twitter (Watkins and Lewis 2014). Watkins and Lewis (2014) looked at professional sport teams within the NBA, WNBA, NFL, NHL and MLS and found that $90 \%$ of the team's mobile applications integrated Twitter, while $65 \%$ provided fans with access to Facebook.

The public access of finding and following Twitter accounts made it an ideal platform to source storylines or dramas. The Twitter account @SevensPhD was created in June 2011 primarily as a mode to source storylines or dramas for data collection and, by October 2012 became a source of case study access, research networking and research brand building.

\section{Selecting Twitter for this research}

There is a particularly good reason for selecting Twitter for this research. Twitter provides an extensive Application Programming Interface (API) that allows access to all public information within Twitter. In contrast, Facebook's privacy issues are more complex than Twitter's, meaning that a lot of status messages are harder to obtain than Tweets, requiring 'open authorization' status from users. Facebook currently stores all data as objects and has a series of APIs, ranging from the Graph and Public Feed APIs to Keyword Insight API. In order to access the properties of an object, its unique ID must be known to make the API call (Wang 2016). Twitter makes it clear to people using it that their posts and profile information are publicly accessible unless they specifically choose to make them all private. This provides a massive database from which data can be extracted. Nevertheless, it should be acknowledged that while it remains comparatively easy to retrieve data from Twitter's API, the nature of that data must also be considered carefully. As Driscoll and Walker (2014) explain: 'The ontology of native Twitter objects is subject to change without warning, and different data sources provide tweets in entirely different formats'. (1747). Similarly, Black 
et al. (2012) suggest that researchers need to have a 'more reflexive stance towards the application programming interfaces twitter provides' (229) saying;

The two key gaps in analysis of social media generally, and Twitter in particular, then are 1)

Each study constructs its own approach to gathering Twitter data and 2) Attempts to explain

the Twitter API through analysis are difficult to verify because the data delivered by API may

be changing over time. (229).

Nevertheless, many researchers are taking advantage of datafication to produce analysis that is informed, nuanced and insightful.

\section{Literature review}

The following review of literature provides a contextual framework for this research. What follows below is a discussion of current research on Twitter followed by a focus on Twitter studies in the area of sport.

\section{Scholarly interest in Twitter}

Academic researchers increasingly utilize Twitter. Veletsianos (2012) identified seven practices of scholars on Twitter:

(1) shared information, resources and media relating to their professional practice;

(2) shared information about their classroom and their students;

(3) requested assistance from and offered suggestions to others;

(4) engaged in social commentary;

(5) engaged in digital identity and impression management;

(6) sought to network and make connections with others; and

(7) highlighted their participation in online networks other than Twitter.

However, although Veletsianos' (2012) research question stated a query for researchoriented practices, the findings were limited to non-research practises outside of sharing information about research projects or asking other scholars about relevant research to their projects. Point 6 - 'sought to network and make connections with others' - could have implicated data access through networks, but Veletsianos did not discuss this aspect.

Ovadia (2009) published one of the first papers to explore Twitter's utility in academic research. Ovadia failed to examine data in the 2009 article, but did point out three superficial functions of Twitter's purported utility in research: (1) as a search tool, particularly of hyper-current information and specific ideas; (2) as access to conversations, particularly to find key people and explore their topics of conversation; and (3) to exchange scholarly ideas under the limitation of 140 characters. In terms of research methods, academics likely utilize the search tool functionality most often, predominantly by searching for keywords and/or hashtags (i.e. \#), judging by the quantity of published works using this method (Lau, Collier, and Baldwin 2012; Sanderson and Gramlich 2016; Small 2011; Sullivan et al. 2012). Second to search tools, academics seem to identify and contact research subjects via access to account profiles and conversations in their research methods (Frederick et al. 2012; Witkemper, Lim, and Waldburger 2012).

Researchers utilize Twitter in detecting dramas that may warrant future investigation. Twitter 'trends' collates tweets about specific incidents, such as scandals breaking and 
unexpected wins. The trends form a basis for classification- or characterization-type research (Cha et al. 2010b; Irani et al. 2010; Lau, Collier, and Baldwin 2012; Naaman, Becker, and Gravano 2011; Rosa, Carvalho, and Batista 2014). Furthermore, researchers perform content analysis on events discoverable via trends (Dykov and Vorobkalov 2013; Sanderson and Gramlich 2016; Small 2011; Vivo 2013). Zhao et al. (2011) derived an innovative method of detecting dramas within a live event that monitored tweets in real time and successfully recognized game events within $40 \mathrm{~s}$. The real-time alerts and masses of instant data available through Twitter provide an exemplary platform for a wide range of research.

In summary, this paper condenses the current literature into an expansion of Veletsianos' Veletsianos' (2012) list of seven practices of scholars who 'engage with' Twitter to include these five additional practices as synthesized from the current paper's case study and the above literature review:

(8) a search tool for almost anything;

(9) a platform for data collection (qualitative data for conversations and content analysis or quantitative data for discovering and investigating trends);

(10) a platform for finding and recruiting research subjects;

(11) an indicator for dramas or incidents relating to one's research field; and

(12) building a personal research brand.

\section{Twitter in sport}

Research surrounding Twitter use in sport has been conducted from a variety of perspectives. Pegoraro (2010) studied athletes and Twitter with a blend of applied research and interpretive research paradigms to analyse professional sports' top athletes use of Twitter. Clavio and Walsh (2014) surveyed Division 1 college sport fans $(n=1036)$ and found that social media should not be viewed holistically due to distinct variability between what attracts fans to Facebook and Twitter. Shockley (2010) utilized content analysis to examine professional athletes' Twitter posts. Gibbs, O’Reilly, and Brunette (2014) studied the fans of eight Canadian Football League teams and their followers on Twitter using content analysis of the public tweets followed by an online survey and semi-structured interviews to identify what motivates the Twitter followers of these particular teams. Frederick, Burch, and Blaszka (2013) performed a content analysis on tweets emitted from @London2012 and tweets containing the hashtag \#London2012. They found that there is no agenda presence on Twitter; the main topic of focus is not transferred to the Twitter audience (Frederick, Burch, and Blaszka 2013).

Clavio, Walsh, and Vooris (2013) performed qualitative interviews with Indy Car series drivers asking questions related to their Twitter usage. Armstrong, Delia, and Giardina (2016) extended how sport teams use Twitter by analysing the Twitter account of the Los Angeles (LA) Kings @LAKings. Johns (2015) conducted a survey that explored how sport fans use Twitter and how Twitter engagement with sport organizations might affect their overall identification to a team. Hull (2014) explored how professional golfers participating in the Masters tournament used Twitter during the week of the event. Basing the research in self-presentation theory, the author conducted a content analysis of 895 tweets by 39 golfers.

Lee (2015) utilized a case study approach using content analysis of the Twitter activity of the Ottawa Senators of the National Hockey League, and their followers. Schultz (2015) 
analysed the use of social media in the National Football League (NFL), the Australian Football League (AFL), NCAA - Football and the Australian minor leagues. Findings illustrated that the NFL utilized Twitter more frequently than the AFL. The NFL also Tweeted more frequently than the NCAA. However, the NCAA utilized Twitter more than the Australian minor leagues. Finally, the AFL was more efficient in Twitter use than the Australian minor leagues. Miller (2016) explored the impacts and influence of Twitter within two National Governing Bodies of sport to evaluate their promotional methods. Quantitative method approach was used to obtain raw data from two sporting National Governing Bodies using Twitter pages. A qualitative content analysis coding framework was then used to discover the use of promotional language to enhance participation.

These studies indicate a diversity of approaches to the evolving area of Twitter studies. It is suggested that this research adds to this increasing research profile and provides new understanding of the role of Twitter in the sport ecosystem and the sport of Sevens in particular.

The original doctoral research on which this current paper is based explored organizational change occurring within rugby national governing bodies in response to Olympic inclusion (Corbett et al. 2015). The personal research brand, established mainly though Twitter, provided the clout and networks for a postdoctoral investigation of Sevens high performance ecosystems funded by rugby's international federation, World Rugby (Corbett, O'Brien, and Gowthorp 2015; O'Brien, Corbett, and Gowthorp 2015). Although the focal academic engaged with the practices mentioned in the literature review, this case study delves into the four additional major uses of Twitter proposed by the authors: (1) a warning system for dramas or incidents relating to one's research field; (2) data collection; (3) finding and recruiting research subjects; and (4) building a personal research brand.

\section{Research design}

In an overview of the use of Twitter for research, Burgess and Bruns (2012) say that data from Twitter require 'new methodological choices in the processing and analysis of such large datasets on mediated social interaction' (1). Traditionally, there have been two main approaches to researching in social media, quantitative and qualitative (Page et al. 2014). Quantitative research can provide a picture of communication that is in a sense 'beyond naked eyes' (Page et al. 2014, p. 51). A flow of information can be captured and visualized at a meta-level in geographical or temporal terms. According to Page et al., this research approach is useful in tracing 'overarching trends which relate to macro-level perspectives' (Page et al. 2014, 51). They suggest however that qualitative studies can also make a significant contribution in that they have the potential to explain the ways in which instances contribute to emerging trends, and to explore the complex ways in which meanings interrelate around particular information and values (Page et al. 2014).

As the primary objective of this research was to identify and extract important information from Twitter about Sevens, the research design chosen used a mixed method approach that combined qualitative and quantitative analysis methods. Mixed method has been growing in popularity among Twitter researchers because it allows researchers to gain a deeper understanding about a situation while being able to analyse a large of data-set at the same time (Bruns and Stieglitz 2013). Combining both of these methods allows Twitter researchers to draw from the strength of both research methods. The qualitative approach included 
performing content analysis on Sevens tweets by evaluating them manually. The quantitative approach included using an off the shelf software package for analysis.

The research approaches utilized in social media research include, but are not limited to, case studies, search and traffic analysis, sentiment analysis and reputation management, population and demographic studies, online target analysis and psychographics and predictive analysis. While many methods share similarities, they all differ in one or two ways. Hale (2016) completed a review of social media research approaches as part of her doctoral studies. Table 2 gives a brief overview of some of these methodologies, and their defining characteristics (Hale 2016).

Table 2. Research approaches for social media.

\begin{tabular}{|c|c|c|c|c|}
\hline Methodology & Data sources & Accessibility of data & $\begin{array}{l}\text { Number of } \\
\text { sources of } \\
\text { data }\end{array}$ & Method of analysis \\
\hline Case studies & $\begin{array}{l}\text { Multiple data } \\
\text { sources }\end{array}$ & $\begin{array}{l}\text { Primarily non-public, } \\
\text { some public }\end{array}$ & Multiple & $\begin{array}{l}\text { Gather multiple data sources about an } \\
\text { event. Analyse and explain what hap- } \\
\text { pened in a particular event }\end{array}$ \\
\hline Search analysis & $\begin{array}{l}\text { Search data } \\
\text { information }\end{array}$ & Primarily non-public & Single & $\begin{array}{l}\text { Use data provided through internally } \\
\text { installed server software or external } \\
\text { analytics tools to understand user } \\
\text { behaviour }\end{array}$ \\
\hline Traffic analysis & Traffic data & $\begin{array}{l}\text { Primarily non-public, } \\
\text { some public }\end{array}$ & Single & $\begin{array}{l}\text { Use data provided through internally } \\
\text { installed server software or external } \\
\text { analytics tools to understand user } \\
\text { behaviour }\end{array}$ \\
\hline $\begin{array}{l}\text { Sentiment } \\
\text { analysis }\end{array}$ & $\begin{array}{l}\text { Social media, } \\
\text { user-generated } \\
\text { content }\end{array}$ & Primarily public & Single & $\begin{array}{l}\text { Gather user-generated content, analyse } \\
\text { it using a list of words that match senti- } \\
\text { ment, determine if sentiment is positive }\end{array}$ \\
\hline $\begin{array}{l}\text { Reputation } \\
\text { management }\end{array}$ & $\begin{array}{l}\text { Social media, } \\
\text { user generated } \\
\text { content }\end{array}$ & Primarily public & Multiple & $\begin{array}{l}\text { Identify places online with positive and } \\
\text { negative sentiment. Develop a solution } \\
\text { to try to change that }\end{array}$ \\
\hline $\begin{array}{l}\text { Content } \\
\text { analysis }\end{array}$ & $\begin{array}{l}\text { Social media, } \\
\text { user-generated } \\
\text { content }\end{array}$ & Primarily public & Single & $\begin{array}{l}\text { Gather relevant content. Study it using } \\
\text { analysis such as discourse analysis }\end{array}$ \\
\hline $\begin{array}{l}\text { Usability } \\
\text { studies }\end{array}$ & Website & Primarily non-public & Single & $\begin{array}{l}\text { Identify tools that can be used to under- } \\
\text { stand specific consumer behaviour. } \\
\text { Use the methodology appropriate for } \\
\text { the chosen tool. This could include eye } \\
\text { tracking glasses, pointer analysis and } \\
\text { click analysis }\end{array}$ \\
\hline $\begin{array}{l}\text { Interaction and } \\
\text { collaboration } \\
\text { analysis }\end{array}$ & $\begin{array}{l}\text { Social media, } \\
\text { user-generated } \\
\text { content }\end{array}$ & Primarily public & Single & $\begin{array}{l}\text { Gather a data-set showing the relation- } \\
\text { ship between two concepts. This can } \\
\text { be supplemented with some content } \\
\text { analysis }\end{array}$ \\
\hline $\begin{array}{l}\text { Relationship } \\
\text { analysis }\end{array}$ & $\begin{array}{l}\text { Social media, } \\
\text { user-generated } \\
\text { content }\end{array}$ & Primarily public & Single & $\begin{array}{l}\text { Gather a data-set showing the relation- } \\
\text { ship between two things }\end{array}$ \\
\hline $\begin{array}{l}\text { Influencer } \\
\text { analysis }\end{array}$ & $\begin{array}{l}\text { Social media, } \\
\text { user-generated } \\
\text { content }\end{array}$ & Primarily public & Single & $\begin{array}{l}\text { Gather a data-set showing the relation- } \\
\text { ship between two things. Try to identify } \\
\text { critical nodes }\end{array}$ \\
\hline $\begin{array}{l}\text { Population } \\
\text { study }\end{array}$ & $\begin{array}{l}\text { Social media, } \\
\text { user-generated } \\
\text { content, surveys }\end{array}$ & $\begin{array}{l}\text { Primarily public, } \\
\text { some non-public }\end{array}$ & Single & $\begin{array}{l}\text { Define community. Gather data regarding } \\
\text { its size and characteristics }\end{array}$ \\
\hline $\begin{array}{l}\text { Demographic } \\
\text { study }\end{array}$ & $\begin{array}{l}\text { Social media, } \\
\text { user-generated } \\
\text { content, surveys }\end{array}$ & $\begin{array}{l}\text { Primarily public, } \\
\text { some non-public }\end{array}$ & Single & $\begin{array}{l}\text { Define community. Gather data regarding } \\
\text { its size and characteristics }\end{array}$ \\
\hline Psychographics & $\begin{array}{l}\text { Survey data, } \\
\text { consumer } \\
\text { information }\end{array}$ & $\begin{array}{l}\text { Primarily non-public, } \\
\text { some public }\end{array}$ & Multiple & $\begin{array}{l}\text { Get off Internet data about consumer } \\
\text { characteristics from a provider of this } \\
\text { information or get it yourself }\end{array}$ \\
\hline
\end{tabular}




\section{Case study methodology}

This research methodology chosen followed a case study approach. Researchers often undertake case studies on social media to measure the effectiveness of specific actions taken by an organization. Bronwyn et al. (2005) state that case studies 'typically examine the interplay of all variables in order to provide as complete an understanding of an event or situation as possible' (p.12). They further suggest that this type of comprehensive understanding is arrived at 'through a process known as thick description, which involves an in-depth description of the entity being evaluated, the circumstances under which it is used, the characteristics of the people involved in it, and the nature of the community in which it is located' (13).

The doctoral dissertation upon which this paper was based occurred between 2011 and 2015, with Twitter being utilized as a research tool from June 2011 and continued past the dissertation submission date, inclusive of the writing of this paper. Specific details on how Twitter was utilized are discussed in the next section.

\section{The case study}

Research followed a case study approach to delve into the four major uses of Twitter in the sport of Sevens: (1) an indicator for dramas or incidents; (2) a platform for data collection; (2) a platform for finding and recruiting research subjects; and (4) building a personal research brand. These uses were not chosen before the research began, but rather unfolded organically as the researcher gradually engaged and had success with Twitter as a research tool.

\section{Twitter as an indicator for dramas or incidents}

Twitter has become a recognized social media platform in the sport industry, as most sporting organizations, such as national governing bodies, have adopted Twitter to communicate with consumers and stakeholders (Smith and Stewart 2010). Research by Chadwick et al. (2010) discussed the integration of Twitter into the strategic operations of sporting organizations.

The first researcher initially created a Twitter account to source incidents of drama that may signal organizational change. This was done in two ways. First, user accounts of rugby national governing bodies, administrators (e.g. CEOs, World Rugby managers), Sevens coaches, Sevens athletes and Sevens-specific media outlets were followed. Following a user account collects each of their 'tweets', or microblogs, onto one webpage called a Twitter feed. Routine checks of the feed occurred every morning for incidents of drama related to the thesis. For example, the news of USA Rugby paying players for the first time in history broke on Twitter (see Figure 1). Information like this critical incident was noted for sequence and then further researched.

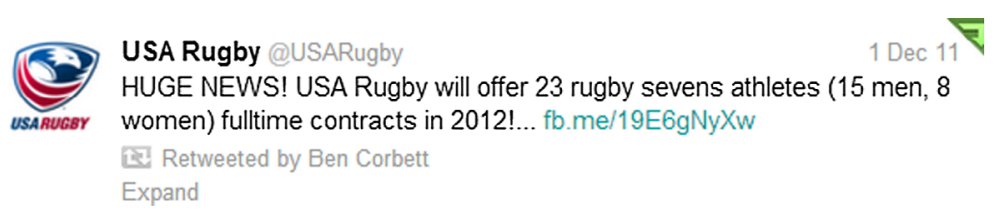

Figure 1. Example tweet from USA Rugby regarding player specialization. 
Table 3. Hashtag keywords searched for this study.

\begin{tabular}{ll}
\hline General Sevens and rugby: & \#Sevens, \#rugby, \#rugbySevens, \#Rugby7s, \#7s \\
Olympics and rugby: & \#OlympicRugby, \#Olympics + \#Sevens, \#rio2016 \\
Case studies: & \#USASevens, \#SASevens, \#KenyaSevens, \#AussieSevens, \#AUSSevens \\
Women's rugby: & \#womensrugby, \#womensSevens \\
\hline
\end{tabular}

Second, a 'hashtag' search was conducted on a regular basis, at least once a month and often weekly. Hashtags (the \# symbol) allow categorization of keywords or topics (Twitter 2016). When multiple users tweet about a particular phenomenon, they put a '\#' in front of the word or short phrase without spaces. The hashtag is the beginning of 'searchable talk' which is 'an online conversation where people actively render their talk more findable' (Zappavigna 2011,25). According to Zappavigna (2011), the hashtag 'presupposes a virtual community of interested listeners who are actively following this keyword or who may use it as a search term' (7). For example, when users discuss Sevens they may use '\#Sevens' or '\#7s' so that others can search and find their tweet. Users can search for \#Sevens and a feed of all tweets containing that keyword will appear. Table 3 lists the hashtag keywords searched for this study. Hashtags are ultimately used to increase the 'loudness' of ones' tweet; the likelihood that they will be seen by others and thereby increasing the 'probability that a user's production [of tweets] over time will be actively "followed" by others' (Zappavigna 2011,21). If enough users tweet with the same hashtag, then that topic will 'trend' or appear automatically on the 'Trends' blog of Twitter, making it easy for users to view popular topics. The Trends blog (worldwide and regional) was accessed periodically to notice any new keywords or popular trends in Sevens, and Sevens trends usually occurred during large critical incidents such as a head coach change.

What the data suggest is that there exists a global Sevens social network. Huberman, Romero, and $\mathrm{Wu}$ (2009) noted that although the 'standard definition of a social network embodies the notion of all the people with whom one shares a social relationship; in reality people interact with very few of those "listed" as part of their network'. As such, they distinguished between a social network represented by the Twitter API and the social network that matters to the user in question. This, Huberman, Romero, and Wu (2009) suggest, implies that reciprocated attention is an important driver of activity on Twitter. They also conclude that Twitter users have a small number of friends compared to their follower-following connections. 'This implies the existence of two different networks: a very dense one made up of followers and followees, and a sparser and simpler network of actual friends' (Huberman, Romero, and Wu 2009). The ability to identify Twitter users in this research and to track the flow of information between them has framed a good understanding of the key issues which were trending in the global Sevens community at any particular moment.

\section{Twitter as a platform for data collection}

Collecting Twitter data involves engaging with the Twitter API. http://dev.twitter.com The Twitter API is the single entry point for sending queries to the Twitter databases and for returning data from those queries. There are two parts to the API, however. One is the Search or REST API, which exists at https://api.twitter.com/1.1/search/, the other is the Streaming API, which requires an ongoing connection through https://stream.twitter.com/1.1/. In order to establish a connection, both require an exchange of authentication codes, which must be generated by registered Twitter users. Twitter restricts access to its API through 
two methods. On the one hand, Twitter limits the rate at which individuals can send queries to the Search and Streaming APIs.

Tweets were collected using a free-to-use online script. For the purposes of this piece of research, the search term used was 'from:@username OR to:@username', where the sequence @username was replaced by a genuine user name for each data query, which facilitated the collection of messages both sent and received by respective users. A 'hashtag' search was conducted on a regular basis, at least once a month and often weekly. Hashtags (the \# symbol) allow categorization of keywords or topics (Twitter 2016).

Tweets analysed were sent during the period June 2012 to September 2014. User accounts of Sevens national governing bodies, administrators (e.g. CEOs, World Rugby managers), Sevens coaches, Sevens athletes and Sevens-specific media outlets were followed.

This study therefore considers only a particular user profile, and does not offer a holistic view of Twitter. A further prerequisite for inclusion was the apparent use of English as the primary language of communication on their Twitter feed; no attempts were made to choose only native speakers, and tweets containing foreign languages were included within the data. These were excluded from content analyses, but were included in the generic quantitative analyses. As Twitter's light weight framework does not oblige users to provide demographic information upon registering, such considerations played no part when selecting potential participants. The resulting corpus contains a total of 11,187 tweets.

Twitter Metadata are structured data provided by Twitter that allows access to feature objects that may or may not be visible directly from tweets. According to Dwoskin (2014), each tweet associates with 150 different metadata, which include commonly visible metadata such as retweet counts and hashtags, to metadata that can only be accessed through an API, such as location metadata. A number of studies have used Twitter metadata to identify key moments in communicative activities because metadata can go beyond the contents of the tweet to identify important information (Burks, Miller, and Zadeh 2014; Leetaru et al. 2013).

Twitter feeds were used as data themselves. NVivo software allowed collection and analysis of Twitter feeds through its NCapture plugin for the Chrome browser. NVivo's Twitter analysis capabilities that the researcher leveraged include categorization, geo-visualization, cluster analysis and charting (e.g. account mentions as seen in Figure 2), time charts and content coding. For example, the increase in tweets and certain hashtag keywords provided evidence of growth and globalization of Sevens. Tracking the number of Sevens-specific user accounts and hashtags (e.g. \#Sevens, \#Sevens7s) across different global regions signalled where and when Sevens was developing or growing in popularity. These data assisted in both describing the Olympic impact on various regions or nations and in deciding what national governing bodies might have enough data to provide a rich case study.

\section{Twitter as a platform for finding and recruiting research subjects}

Case study access unexpectedly occurred while using Twitter. Many coaches and administrators would not reply to emails; however, some were responsive on Twitter. For example, the Kenya Sevens Head Coach responded to a direct tweet to him, and the result was a private message through Twitter that produced a meeting at the Gold Coast Sevens tournament in October 2012, leading to him becoming a gatekeeper to Kenya Sevens Union (see Figure 3). Additionally, the Kenyan High Performance Director and a board member were not available during the visit to Kenya for an interview, and they were engaged via Twitter in August 2013 that led to Skype interviews. Furthermore, the South Africa and USA Sevens 


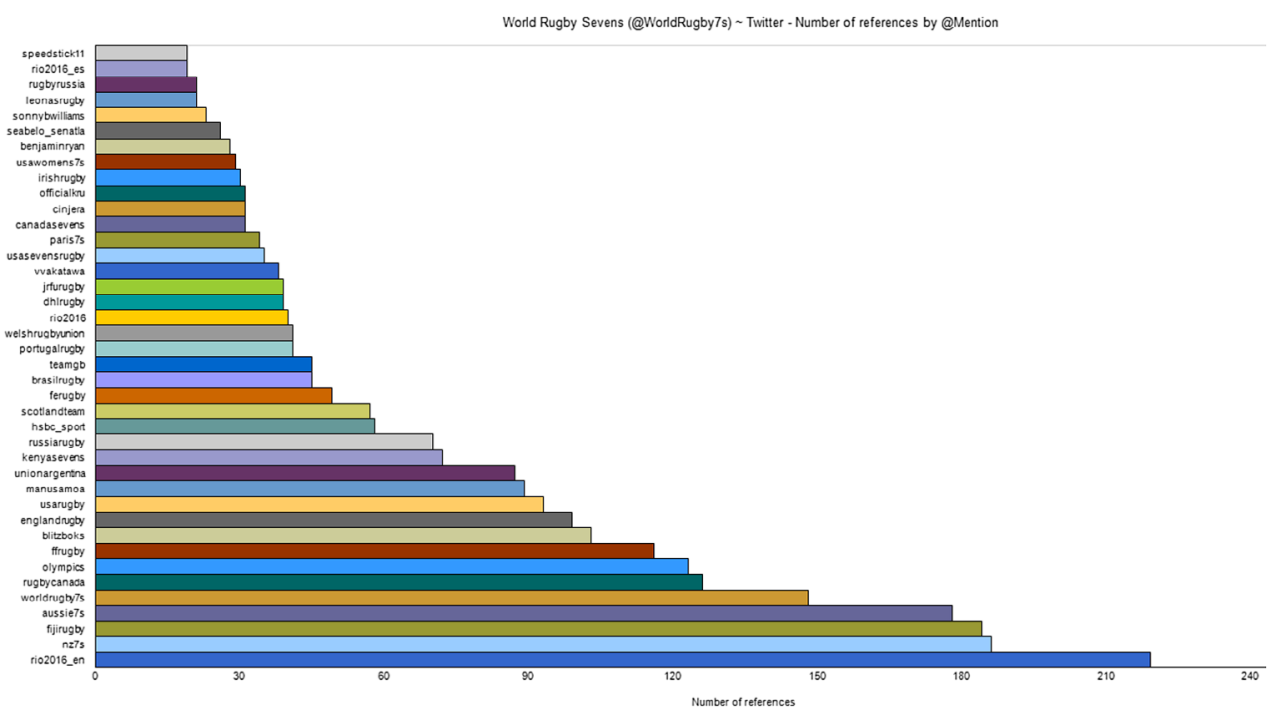

Figure 2. Example chart form NVivo on number of Twitter account mentions by @WorldRugby7s.
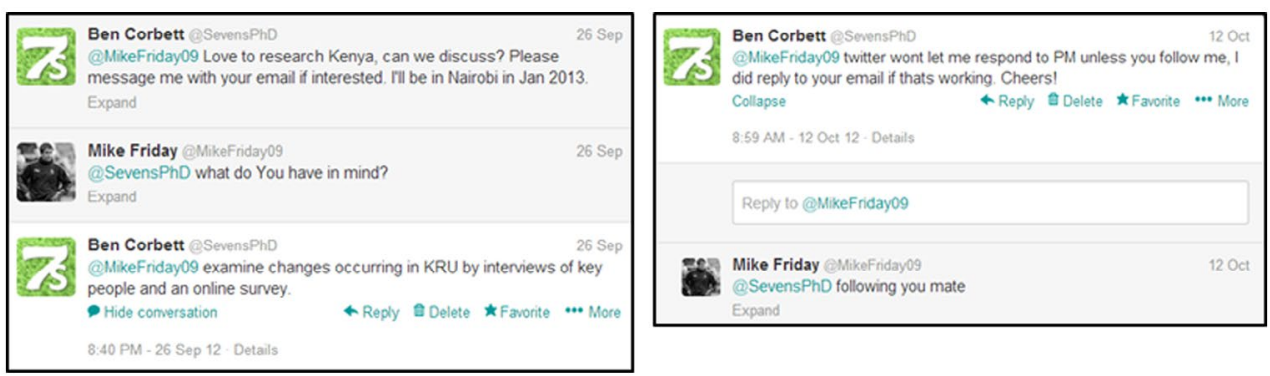

Figure 3. Tweets to engage Kenya as a case study.

Coaches were primarily contacted via Twitter private messages, and led to additional access beyond what was granted through other NGB sources.

Sometimes access to cases was unattainable; however, the attempt raised awareness of the research project. The former England Sevens Coach engaged via Twitter with initial interest in May 2012, but finally denied case access to England in October 2012 as seen in Figure 4. However, communication via Twitter continued with him in an attempt to build trust for access to future research, and succeeded when he became the head coach of Fiji in 2013 and agreed to interview for the postdoctoral study in 2015.

As the @SevensPhD account attracted more followers through building a research and knowledge sharing reputation (discussed in the next section), national governing body administrators and World Rugby managers took notice. Occasionally, those managers contacted @SevensPhD to ask questions about the research and to set meetings for discussion in more detail. These meetings allowed trust building for data access. For example, here is a quote from an email from a high-level World Rugby staff member:

I am sure you will be in the Gold Coast so can we catch up more formally then as your research is gaining a lot of interest - thanks to Twitter although I have to say there are some graphs etc. which I don't fully understand. 

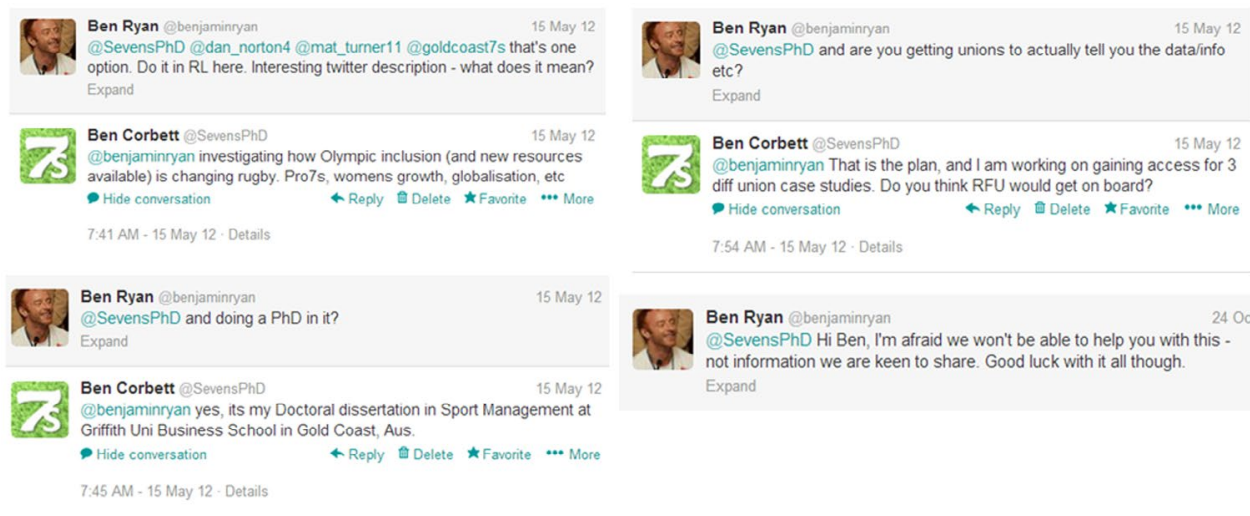

Ben Ryan

240

Q Expand

Figure 4. Tweets engaging with Ben Ryan, England Sevens Head Coach.

The email response led to a meeting at the 2013 HSBC World Rugby Sevens Series tournament in Australia, with follow-up meetings and interviews with several key World Rugby and national governing body managers at the 2013 World Rugby Conference in Dublin, Ireland. The meetings during the World Rugby Conference provided the interest from World Rugby to fund the postdoctoral study in Sevens' high-performance ecosystems, and the network built through Twitter provided recognizability for interviewing managers, coaches and players from 32 countries.

\section{Twitter to build a research brand}

Twitter enabled the researcher to build a research brand. As with case study access, there was no initial intent to build a research brand using Twitter. This occurred naturally after the @SevensPhD account was created. In the beginning, only retweets of significant dramas (press releases) were posted to the @Sevens $\mathrm{PhD}$ micro-blog primarily as a way to save and sort the dramas for later data consideration. The retweeting of dramas instigated new followers of @SevensPhD. Then, after initial data were collected in December of 2012, the @SevensPhD account began to build research credibility and legitimacy by posting data, as seen in Figure 5. Note Murray Barnett, World Sevens Head of Commercial, Marketing and Broadcast and Mike Friday, former Head Coach of Kenya Sevens and current Head Coach of USA Sevens engaging with the tweet. Figure 6 illustrates engagement ( 40 retweets, 53 likes, 22 new followers) from a broad range of the public figures with simple data. The engagement is not extraordinary when compared to larger fields, but Sevens has a narrow following compared to mainstream sport, and an account focused on researching Sevens would not be expected to have substantial following. However, the resulting credibility established trust among some national governing body and World Rugby research participants, enabling further data collection as exemplified in the above section.

\section{Ethical considerations}

According to Page et al. (2014), how to define ethical conduct in researching social media is not straightforward. Firstly, the use of social media data creates a number of obligations towards the company which owns that data and the platform on which the data are then 


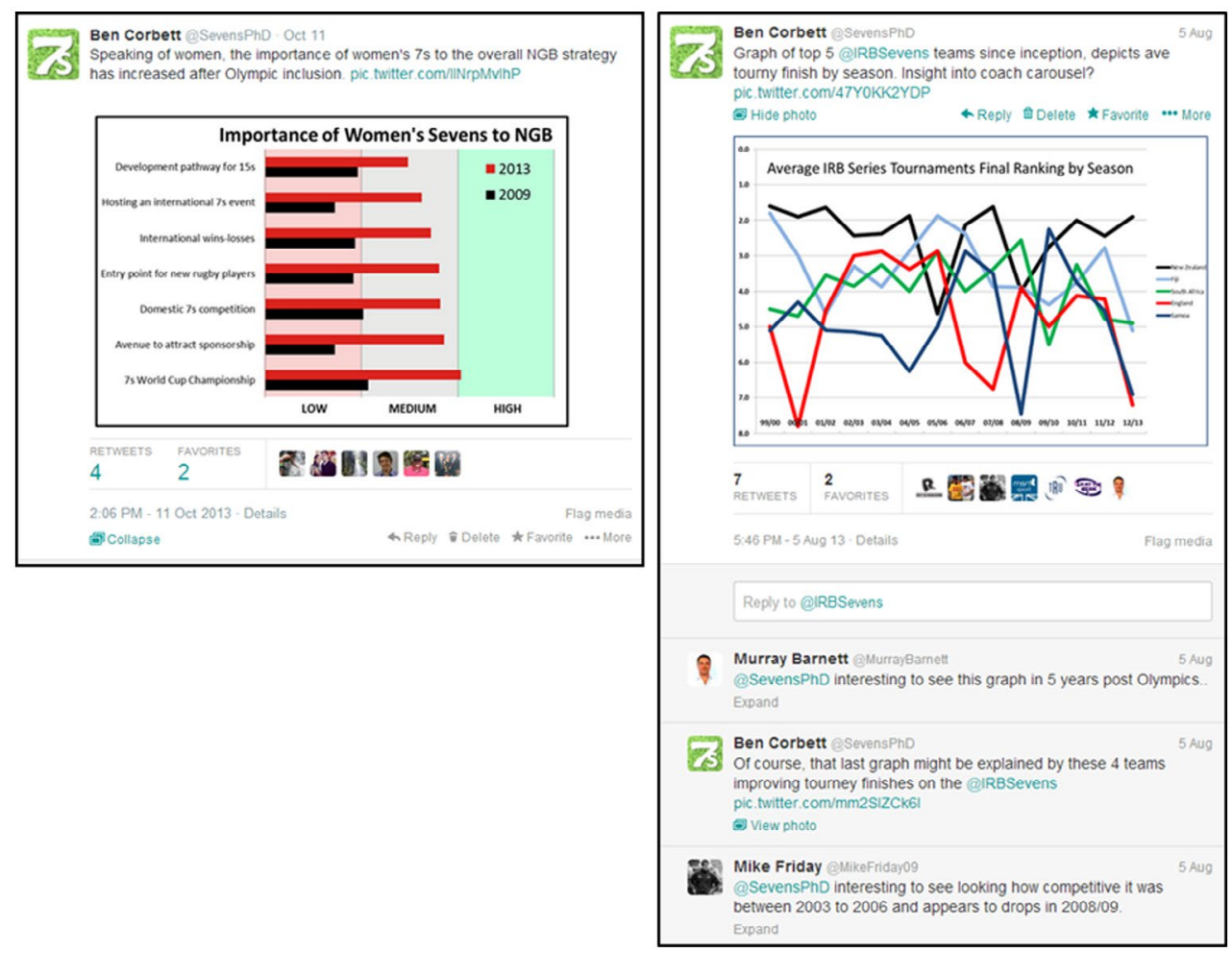

Figure 5. Example of research data tweet from @SevensPhD.

created. The extent of these obligations is still evolving. In recognition of their increasing use in research many of the organizations have developed specific 'Terms of Service' [TOS] which are accessible on each organization's website. Many of these organizations also provide specific technical interfaces for the accessing of data, known as 'Application Programming Interfaces' [API], which allow them to both monitor and set limits on it (a typical Twitter researcher will, for example, only be able to access $1 \%$ of the material published on Twitter on any given day) (Hale 2016).

Hale (2016) indicates that beyond obligations to the data owners, social media researchers also have a series of obligations towards the individuals who originally created that data. Hale (2016) points out that law in this area is developing but also complicated by international legal boundaries. In ethical terms, researchers face the problem that ethical participation in a social media study based around the idea of informed consent is essentially impossible both because of the number of social media users as well as the practical challenge of contacting them. It could be argued that individuals are theoretically aware when they create social media content that it may be both made public and used for other purposes. In practice, few will have considered that it might one day become the object of a research project. Twitter is an unquestionably public platform and upon subscribing, users must agree to terms of service (Twitter 2016) which make this abundantly clear. Nevertheless, most contemporary researchers base their research ethics around the idea of 'minimizing harm' (Page et al. 2014). 


\section{Olympic effect: Google trends for searching "rugby sevens" - Worldwide, Past 5 years -}

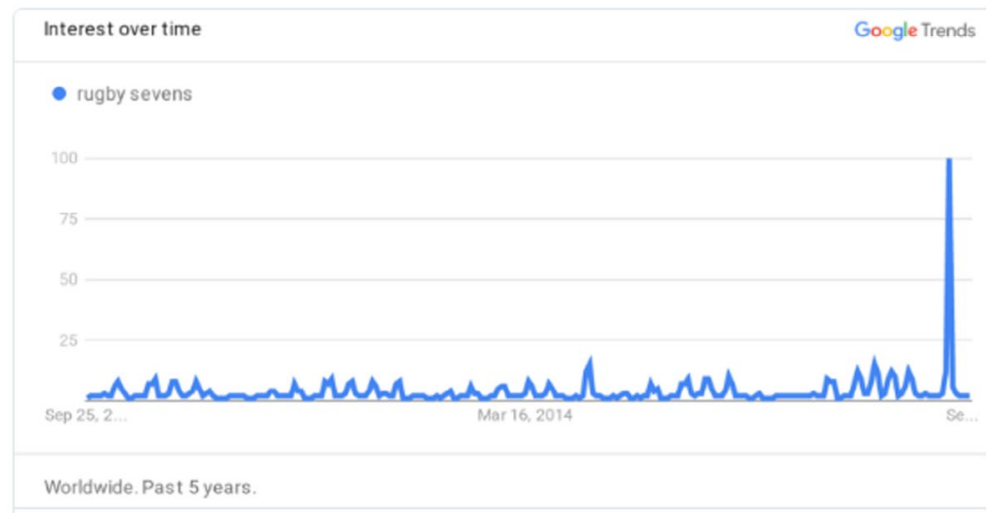

\section{Google Trends}

See interest over time on Google Trends for rugby sevens - Worldwide, Past 5 years.

google.com

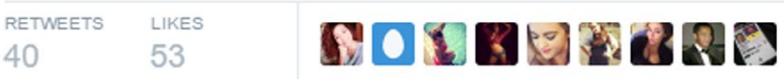

3:48 PM - 21 Sep 2016

Figure 6. More recent example of engagement with @SevensPhD tweet.

Although social media research is unlikely to cause physical harm to the participants in the way medical research might cause, Page et al. (2014) warn that 'harms to participants may appear in forms that are not immediately tangible but nonetheless may be significant' (59). For instance, in the present study that attends to data posted on Twitter, unethical conduct may occur by 'quoting material which might overtly or inadvertently expose some part of a person's identity or activities that they had wished to keep private' (59).

Page et al. (2014) also discuss that ethical considerations need to pay attention to 'whether a researcher considers the object of analysis to be people (their identities, behaviour and interaction) or text (as a decontextualized object)' (59-60). The present study falls into the latter in the sense that focus is not on Twitter users but on their tweeter discourse. It is inevitable that some aspects of the professional identity of the writers are made explicit in the research. It is then important that the research is designed to avoid possible harm that might come by treating posts on social media as a data-set. It is also noted that, as Page et al. mentions, the current regulation of Twitter 'prohibits academics from sharing corpora built from archives of harvested tweets' (63).

In the present study, possible ethical issues are overcome in the following ways. First, the data were collected from official Twitter sites taken from public users only, and from 
accounts that are free-to-view rather than private. It was considered ethically sound to pursue such a line of enquiry, on the basis that user links and any other sensitive information would not be published. Second, in terms of the identity of writers of the data, the selected tweeters all authorized their tweets by providing their identity. It is assumed that all writers in focus tweet as a public practice, although they might mention more private matters occasionally.

\section{Limitations of the research}

We presented an assortment of analytic techniques on Twitter throughout this research that can provide a useful insight on the idiosyncratic behaviour peculiar to Twitter and a foundation for future related work. Despite conducting the research contributions as rigorously as possible, some imitations were inevitable. By electing to use a single social media source (Twitter) we have restricted the demographic of the people contributing to the discussion to Twitter users and the style of their contribution to 140 character tweets. There are many other forms of social media, such as Facebook, that could also be used as 'Open Source Intelligence' and would give access to different types of information and other demographic groups. Restrictions that apply to collection of Twitter data impact the quality of data available for this study. It is difficult to localize tweets which limits our ability to consider regional or national differences in conversations. People use Twitter in languages other than English but our study is limited to English. The restrictions by Twitter on obtaining past tweets means that it is necessary to wait for tweets to be created by people on Twitter before they can be collected as data.

Perhaps the biggest limitation encountered and documented throughout is the ability of this study to generalize findings because of the limited data sample size. A larger data sample size is proposed (future research) for a greater degree of generalization of the findings. Nevertheless, patterns investigated by this study through the limited sample size are of strong theoretical interest for understanding the global Sevens community.

With regard to the specific limitations of the medium, the 140-character format is certainly a Twitter-specific restraint. It is suggested that it need not be seen as a limitation and may even be seen as an advantage. The brevity of messages allows them to be produced, consumed and shared without the need for significant investment in thought, time and effort, which, in turn, engenders a fast and fluid interactive environment (Boyd, Golder, and Lotan 2010).

Twitter is a highly dynamic environment which is perhaps only beginning to settle down after a short embryonic period during which it has grown exponentially. Aside from the character limitation, there is little evidence to suggest that tweets constitute a single written genre, but a hybrid of genre features identified in different text types, from instant messaging to texting and blogging. This an area for future investigation.

\section{Contribution of the study}

Twitter has become its own legitimate and specific genre of communication, and the construction of a tweet has great significance when the goal is to resonate with and influence other users. The ability to generate and sustain discussions on Twitter is of significant strategic importance in sport. This study does not provide a formula for achieving this, 
but contributes to the wider understanding of Twitter and provides a starting point for the planning and implementation of Twitter use in sport.

\section{Directions for future sport research}

This study has revealed that Twitter has become its own legitimate and specific genre of communication, and that the construction of a tweet has great significance when the goal is to resonate with and influence other users. Unlike other social media platforms, Twitter's usage mechanism is unlikely to change significantly in the future. This provides an opportunity for research into the platform to be conducted in such a way that a wider understanding can be reached about its role in sport.

\section{Conclusion}

Twitter is a highly dynamic environment which is perhaps only beginning to settle down after a short embryonic period during which it has grown exponentially. By examining the communicative behaviour of Sevens Twitter users over a 28 -month period, this study has provided an assessment of some of the pertinent linguistic phenomena to be found on the medium. An analysis of the basic habits of Sevens Twitter users showed that tweets are posted with variable frequency, with some using the medium only intermittently, while others utilize it frequently. A macro-level overview of the tweets established that Twitter supports inter-user dialogue and that the @ symbol is used extensively as a strategy to facilitate the coherent exchange of messages, contributing to a highly interactive environment in which interactions can occur frequently and over lengthy periods. It was also found that the retweet function fulfils numerous functions, most notably to contribute to a wider conversation but poses significant challenges in terms of the interpretation of such messages. Retweets often were seen to obscure meaning and raise questions regarding authorship, ownership and conversational fidelity. Hashtag-ing introduced additional levels of complexity in analysis through elliptical commentary.

For the Sevens user, Twitter is no incidental communicative medium; instead, it plays an integral role in many individuals' communicative and social behaviours, and thus constitutes an important and widely used addition to the family of new media technologies. Tweets exhibit high degrees of self-presentation and self-disclosure which engender strong feelings of identification with Sevens.

Even post-research it is important to keep the Twitter account relevant and updated. Although the researcher in this case study shifted research focus temporarily from Sevens, the account continues to focus on the niche sport. This allows the academic to continually engage in almost all of the 12 Twitter practices, and particularly maintain a personal research brand and discover dramas that may merit future research.

\section{Disclosure statement}

No potential conflict of interest was reported by the authors.

\section{ORCID}

Allan Edwards (D) http://orcid.org/0000-0002-1901-9049 


\section{References}

Armstrong, C. G., E. B. Delia, and M. D. Giardina. 2016. "Embracing the Social in Social Media an Analysis of the Social Media Marketing Strategies of the Los Angeles Kings." Communication \& Sport 4 (2): 145-165. doi:10.1177/2167479514532914.

Black, A., C. Mascaro, M. Gallagher, and S. P. Goggins. 2012. "Twitter Zombie: Architecture for Capturing, Socially Transforming and Analyzing the Twittersphere." Paper presented at the Proceedings of the 17th ACM International Conference on Supporting Group Work, Sanibel Island, Florida, USA.

Boyd, D., S. Golder, and G. Lotan. 2010. “Tweet, Tweet, Retweet: Conversational Aspects of Retweeting on Twitter." Paper presented at the 2010 43rd Hawaii International Conference on System Sciences (HICSS), Honolulu, Hawaii, USA.

Bronwyn, B., P. Dawson, K. Devine, C. Hannum, S. Hill, J. Leydens, and M. Palmquist. 2005. Case Studies. http://writing.colostate.edu/guides/research/casestudy/.

Bruns, A., and S. Stieglitz. 2012. "Quantitative Approaches to Comparing Communication Patterns on Twitter." Journal of Technology in Human Services 30 (3-4): 160-185.

Bruns, A., and S. Stieglitz. 2013. "Towards More Systematic Twitter Analysis: Metrics for Tweeting Activities." International Journal of Social Research Methodology 16 (2): 91-108. doi:10.1080/136 45579.2012.756095.

Burgess, J., and A. Bruns. 2012. "Twitter Archives and the Challenges of 'Big Social Data' for Media and Communication Research." M/C Journal 15 (5). ISSN 14412616. Accessed june 16, 2017. http:// journal.media-culture.org.au/index.php/mcjournal/article/view/561

Burks, L., M. Miller, and R. Zadeh. 2014. "Rapid Estimate of Ground Shaking Intensity by Combining Simple Earthquake Characteristics with Tweets." Paper presented at the 10th US Nat. Conf. Earthquake Eng., Anchorage, AK, USA.

Cha, M., H. Haddadi, F. Benevenuto, and P. K. Gummadi. 2010a. "Measuring User Influence in Twitter: The Million Follower Fallacy." Paper presented at the Fourth International AAAI Conference on Weblogs and Social Media, Online, Washington, DC.

Cha, M., H. Haddadi, F. Benevenuto, and P. K. Gummadi. 2010b. "Measuring User Influence in Twitter: The Million Follower Fallacy." ICWSM 10 (10-17): 30.

Chadwick, S., A. Semens, E. Schwarz, and D. Zhang. 2010. Economic Impact Report on Global Sevens Part III: Strategic and Emerging Markets. Retrieved from Coventry University. Coventry, UK.

Clavio, G., and P. Walsh. 2014. "Dimensions of Social Media Utilization among College Sport Fans." Communication \& Sport 2 (3): 261-281. doi:10.1177/2167479513480355.

Clavio, G., P. Walsh, and R. Vooris. 2013. "The Utilization of Twitter by Drivers in a Major Racing Series." International Journal of Motorsport Management 2 (1): 2.

Constantinides, E., and S. J. Fountain. 2008. "Web 2.0: Conceptual Foundations and Marketing Issues." Journal of Direct, Data and Digital Marketing Practice 9 (3: 231-244. doi:10.1057/palgrave. dddmp.4350098.

Corbett, B., D. O’Brien, and L. Gowthorp. 2015. "Efficacy of High Performance Training Regimes in Sevens Sevens." Paper presented at the The World in Union: Sevens's Past, Present, and Future, Brighton, UK.

Corbett, B., J. Skinner, D. O'Brien, and C. Auld. 2015. "Strategic Change in Response to an Environmental Jolt: Sevens and the Olympic Games." Doctor of philosophy, Griffith University.

Driscoll, K., and S. Walker. 2014. "Big Data, Big Questions| Working within a Black Box: Transparency in the Collection and Production of Big Twitter Data." International Journal of Communication 8: 20.

Dwoskin, E. 2014. In a Single Tweet, as Many Pieces of Metadata as There Are Characters. http:// blogs.wsj.com/digits/2014/06/06/in-a-single-tweet-as-many-pieces-of-metadata-as-there-arecharacters/.

Dykov, M. A., and P. N. Vorobkalov. 2013. "Twitter Trends Detection by Identifying Grammatical Relations." Paper presented at the 26th International FLAIRS Conference, St. Pete Beach, Florida, USA.

Frederick, E. L., L. M. Burch, and M. Blaszka. 2013. "A Shift in Set: Examining the Presence of Agenda Setting on Twitter During the 2012 London Olympics." Communication \& Sport 3 (3): 312-333. doi:10.1177/2167479513508393. 
Frederick, E. L., C. H. Lim, G. Clavio, and P. Walsh. 2012. "Why We Follow: An Examination of Parasocial Interaction and Fan Motivations for following Athlete Archetypes on Twitter." International Journal of Sport Communication 5 (4): 481-502. doi:10.1123/ijsc.5.4.481.

Gibbs, C., N. O’Reilly, and M. Brunette. 2014. "Professional Team Sport and Twitter: Gratifications Sought and Obtained by Followers." International Journal of Sport Communication 7 (2): 188213. doi:10.1123/IJSC.2014-0005.

Hale, L. 2016. "Social Response Analysis: Exploring Social Media Demographic Changes in Response to Sporting Event." Doctoral, University of Canberra, Unpublished.

Huberman, B. A., D. M. Romero, and F. Wu. 2009. Crowdsourcing, Attention and Productivity. Social Computing Lab, HP Laboratories, Palo Alto, CA 94304. http://arxiv.org/abs/0809.3030.

Hull, K. 2014. "A Hole in One (Hundred Forty Characters): A Case Study Examining PGA Tour Golfers' Twitter Use during the Masters." International Journal of Sport Communication 7 (2): 245-260. doi:10.1123/IJSC.2013-0130.

Ioakimidis, M. 2010. “Online Marketing of Professional Sports Clubs: Engaging Fans on a New Playing Field." International Journal of Sports Marketing and Sponsorship 11 (4): 2-13. doi:10.1108/ IJSMS-11-04-2010-B002.

Irani, D., S. Webb, C. Pu, and K. Li. 2010. "Study of Trend-stuffing on Twitter through Text Classification." Paper presented at the Collaboration, Electronic messaging, Anti-Abuse and Spam Conference (CEAS), Redmond, Washington, USA.

Johns, J. 2015. “Twitter and Fan Identification: Lessons Learned from Athletic Communication S Work Experience." Master of Arts, Clemson University.

Lau, J. H., N. Collier, and T. Baldwin. 2012. "On-line Trend Analysis with Topic Models:\#\# Twitter Trends Detection Topic Model Online." Paper presented at the COLING, Mumbai, India.

Lee, J. 2015. The Ottawa Senators Twitter Activity and the Effect on Their Followers. Unpublished thesis. Ottowa, CA: Université d'Ottawa/University of Ottawa.

Leetaru, K., S. Wang, G. Cao, A. Padmanabhan, and E. Shook. 2013. "Mapping the Global Twitter Heartbeat: The Geography of Twitter." First Monday 18 (5): 290-307. doi:10.5210/fm.v18i5.4366.

Li, M., E. MacIntosh, and G. Bravo. 2012. International Sport Management. Champaign, IL: Human Kinetics.

Lipsman, A. 2009. What Ashton Vs. CNN Foretold about the Changing Demographics of Twitter. http://www.comscore.com/Insights/Blog/What-Ashton-vs.-CNN-Foretold-About-the-ChangingDemographics-of-Twitter.

Miller, K. J. 2016. "An Investigation into How a Sporting National Governing Body Utilises Twitter as a Promotional Marketing Tool to Enhance Grass Root Participation." Bachelors of Science, Cardiff Metropolitan University.

Naaman, M., H. Becker, and L. Gravano. 2011. "Hip and Trendy: Characterizing Emerging Trends on Twitter." Journal of the American Society for Information Science and Technology 62 (5): 902-918. doi:10.1002/asi.21489.

O’Brien, D., B. Corbett, and L. Gowthorp. 2015. "How Olympic Inclusion Impacts High Performance Training: The Case Study of Sevens Sevens." Paper presented at the European Association of Sport Management, Dublin, Ireland.

Ovadia, S. 2009. “Exploring the Potential of Twitter as a Research Tool." Behavioral \& Social Sciences Librarian 28 (4): 202-205. doi:10.1080/01639260903280888.

Page, R., D. Barton, J. W. Unger, and M. Zappavigna. 2014. Researching Language and Social Media: A Student Guide. New York, NY: Routledge.

Pegoraro, A. 2010. "Look Who's Talking - Athletes on Twitter: A Case Study." International Journal of Sport Communication 3 (4): 501-514. doi:10.1123/ijsc.3.4.501.

Romero, D. M., W. Galuba, S. Asur, and B. A. Huberman. 2011. "Influence and Passivity in Social Media." Paper presented at the Joint European Conference on Machine Learning and Knowledge Discovery in Databases. Athens, Greece.

Rosa, H., J. P. Carvalho, and F. Batista. 2014. "Detecting a Tweet's Topic within a Large Number of Portuguese Twitter Trends." Paper presented at the OASIcs-OpenAccess Series in Informatics, Braganca, Portugal. doi:10.4230/OASIcs.SLATE.2014.185. 
Sanderson, J., and K. Gramlich. 2016. "'You Go Girl!': Twitter and Conversations about Sport Culture and Gender." Sociology of Sport Journal 33 (2): 113-123. doi:10.1123/ssj.2015-0048.

Schultz, A. 2015. "An Analysis of the Utilization of Social Media in Gridiron Football with Direct Comparison to Australian Rules Football in Addition to Respective College and Minor Leagues." Bachelors, Eastern Michigan University.

Shockley, J. 2010. "Unfiltered? A Content Analysis of pro Athletes' 'Twitter' Use." Master in Arts, East Tennessee State University.

Small, T. A. 2011. "What the Hashtag? A Content Analysis of Canadian Politics on Twitter." Information, Communication \& Society 14 (6): 872-895. doi:10.1080/1369118X.2011.554572.

Smith, A. C., and B. Stewart. 2010. “The Special Features of Sport: A Critical Revisit." Sport Management Review 13 (1): 1-13. doi:10.1016/j.smr.2009.07.002.

Sullivan, S. J., A. G. Schneiders, C.-W. Cheang, E. Kitto, H. Lee, J. Redhead, ... P. R. McCrory. 2012. "What's Happening?'A Content Analysis of Concussion-Related Traffic on Twitter." British Journal of Sports Medicine 46 (4): 258-263. doi:10.1136/bjsm.2010.080341.

Twitter. 2016. Using Twitter. https://support.twitter.com/categories/281.

Veletsianos, G. 2012. "Higher Education Scholars' Participation and Practices on Twitter." Journal of Computer Assisted Learning 28 (4): 336-349. doi:10.1111/jca.2012.28.issue-4.

Vivo, J. M. N. 2013. “ ¿ Qué Transparencia Tienen Los Periodistas En Twitter? Tendencias Hacia Un Periodismo Centrado En El Usuario." [How Open Are Journalists on Twitter? Trends towards the End-user Journalism.] Comunicación Y Sociedad 26 (1): 93-114.

Wang, Xinyue. 2016. "Real-time Content Identification for Events and Sub-events from Microblogs." Doctoral, Queen Mary University of London, Unpublished.

Watkins, B., and R. Lewis. 2014. "Winning with Apps: A Case Study of the Current Branding Strategies Employed on Professional Sport Teams' Mobile Apps.” International Journal of Sport Communication 7 (3): 399-416. doi:10.1123/IJSC.2014-0009.

Williams, J., and S. J. Chinn. 2010. "Meeting Relationship-marketing Goals through Social Media: A Conceptual Model for Sport Marketers." International Journal of Sport Communication 3 (4): 422-437. doi:10.1123/ijsc.3.4.422.

Witkemper, C., C. H. Lim, and A. Waldburger. 2012. "Social Media and Sports Marketing: Examining the Motivations and Constraints of Twitter Users." Sport Marketing Quarterly 21 (3): 170-183.

Zappavigna, M. 2011. "Ambient Affiliation: A Linguistic Perspective on Twitter." New Media \& Society 13 (5): 788-806. doi:10.1177/1461444810385097.

Zhao, S., L. Zhong, J. Wickramasuriya, and V. Vasudevan. 2011. Human as Real-time Sensors of Social and Physical Events: A Case Study of Twitter and Sports Games. ArXiv Preprint ArXiv:1106.4300. 